\title{
Ecto-calreticulin is essential for an efficient immunogenic cell death stimulation in mouse melanoma
}

\author{
by Paola Giglio and colleagues, Genes and Immunity, October 4, 2018
}

\section{Thomas Brunner ${ }^{1}$}

Published online: 4 January 2019

C) Springer Nature Limited 2018

With the Nobel Prize in Physiology award to James P. Allison and Tasuku Honjo for their research of immune check points, and the use of immune checkpoint inhibitors in the clinics, tumor immunology has entered a new age. Similarly, many pharmaceutical companies are massively investing into therapies with chimeric antigen receptor (CAR)-expressing $\mathrm{T}$ cells. It is now generally accepted that unleashing and/or harnessing the patient's own immune system may result in positive therapeutic effects at least in some types of tumors. While the idea that our immune system is potentially capable of controlling the expansion of transformed cells and thereby the development of cancer, is quite old-it was already proposed by Paul Ehrlich and others at the beginning of the twentieth century-its scientific confirmation and in particular clinical application remained vague until recently. Though there has been a lot of experimental evidence that tumors may elicit specific immune responses, and that antitumor immune cells may indeed contribute to the control of tumor growth and patient's fate, therapeutic strategies aiming at stimulating the patient's own immune system by cancer vaccination remained largely unsuccessful. Part of the challenges has been and remains that tumor cells are largely identical to normal host cells, and minor differences in gene expression and antigenic determinants (tumor-associated antigens) are often insufficient to trigger a curative immune response against the tumor. Thus, while the availability of novel technologies, such as MHC tetramers, allowed the detection of tumor antigen-specific $\mathrm{T}$ cells in melanoma patients after vaccination with tumor antigen $[1,2]$, the induction of

Thomas Brunner

thomas.brunner@uni-konstanz.de

1 Department of Biology, University of Konstanz, Konstanz, Germany tumor-specific immune responses appeared to be unparalleled by therapeutic success.

Clearly, the availability of tumor-specific $\mathrm{T}$ cells is not sufficient to kill tumor cells, but the tumor-controlled unresponsiveness of cytotoxic $\mathrm{T}$ cells needs to be unleashed. In this regard, the development of immune checkpoint inhibitors, such as anti-PDL1 and anti-CTLA4, has been a major breakthrough in tumor immunology and tumor therapy (reviewed in refs $[3,4]$ ). Yet, while many patients are successfully treated with these biologicals, leading to tumor remission and increased survival, sadly, others remain unresponsive to checkpoint inhibitors. Thus, clearly, other tumor- and immune cell-specific aspects control and prevent efficient antitumor immune responses.

This is where immunogenic cell death (ICD) may contribute to an increased response of tumor patients. While necrotic cell death was generally considered to be of inflammatory nature, and apoptotic cell death immunologically silent, it is now clear that depending on how cell death is induced, immunostimulatory factors may be released by dying tumor cells, which enhance the activation of immune cells and thus act as adjuvants. Treatment of tumor cells by certain chemotherapeutic drugs, such as anthracyclins, oxaliplatin and bortezomib, or radiotherapy, results in a form of cell death, i.e., ICD, which causes the release of cellular components with high immunostimulatory activity (reviewed in refs $[5,6]$ ). While the term "danger-associated molecular patterns" (DAMPs) was already defined some time ago by Polly Matzinger [7, 8], the identification of these immunostimulatory molecules released from dying cells, such as extracellular ATP, HMGB1, and calreticulin, is now advanced. The specific release of such DAMPs by tumor cells may thus contribute to the induction of a protective antitumor immune response.

As with most things in life, though, successful tumor therapy is not as simple, and the plethora of tools and strategies tumor cells develop to evade destruction by the immune 
system appears almost endless. The prevention of DAMP release and associated lack of immune stimulation was investigated in a recent article by Paola Giglio et al. [9]. They find that lack of calreticulin exposure in anthracyclin-treated dying melanoma cells largely prevents a successful control of tumor growth by the immune system. Understanding the molecular basis of the regulation of successful DAMP release and/or exposure on plasma membranes of dying tumor cells may thus help to enhance the immunogenicity of tumors and boost the patient's own immune defense. This paper is the Editor's pick as negative findings can stimulate new directions.

\section{Compliance with ethical standards}

Conflict of interest The authors declare that they have no conflict of interest.

Publisher's note: Springer Nature remains neutral with regard to jurisdictional claims in published maps and institutional affiliations.

\section{References}

1. Pittet MJ, Valmori D, Dunbar PR, Speiser DE, Lienard D, Lejeune F, et al. High frequencies of naive Melan-A/MART-1- specific CD8(+) $\mathrm{T}$ cells in a large proportion of human histocompatibility leukocyte antigen (HLA)-A2 individuals. J Exp Med. 1999;190:705-15.

2. Coulie PG, Karanikas V, Colau D, Lurquin C, Landry C, Marchand $\mathrm{M}$ et al. A monoclonal cytolytic T-lymphocyte response observed in a melanoma patient vaccinated with a tumor-specific antigenic peptide encoded by gene MAGE-3. Proceedings of the National Academy of Sciences of the United States of America 2001;98: 10290-5.

3. Festino L, Vanella V, Trojaniello C, Ascierto PA. Selecting immuno-oncology-based drug combinations - what should we be considering? Expert Rev Clin Pharmacol. 2018;11:971-85.

4. Raschella G, Melino G, Gambacurta A. Cell death in cancer in the era of precision medicine. Genes Immun. 2018.

5. Cruickshank B, Giacomantonio M, Marcato P, McFarland S, Pol J, Gujar S. Dying to be noticed: epigenetic regulation of immunogenic cell death for cancer immunotherapy. Front Immunol. 2018;9:654.

6. Galluzzi L, Buque A, Kepp O, Zitvogel L, Kroemer G. Immunogenic cell death in cancer and infectious disease. Nat Rev Immunol. 2017;17:97-111.

7. Gallucci S, Matzinger P. Danger signals: SOS to the immune system. Curr Opin Immunol. 2001;13:114-9.

8. Matzinger P. Tolerance, danger, and the extended family. Annu Rev Immunol. 1994;12:991-1045.

9. Giglio P, Gagliardi M, Bernardini R, Mattei M, Cotella D, Santoro $\mathrm{C}$ et al. Ecto-Calreticulin is essential for an efficient immunogenic cell death stimulation in mouse melanoma. Genes Immun. 2018. 